\title{
Hormetic effect of gamma irradiation under salt stress condition in Phaseolus vulgaris
}

\author{
Kamile Ulukapi ${ }^{{ }^{*}}$ \\ ${ }^{1}$ Akdeniz University, Vocational High School of Technical Sciences, Department of Plant and Animal Production, 07059, Antalya, \\ Turkey. "Corresponding author (kamileonal@akdeniz.edu.tr).
}

Received: 29 December 2020; Accepted: 17 March 2021; doi:10.4067/S0718-58392021000300256

\begin{abstract}
Common bean (Phaseolus vulgaris L.) is widely grown around the world and sensitive to stress conditions. Accumulation and degradation of amino acids are informative indicators of plant tolerance. In this study, the vegetative growth and amino acid profiles of two common bean cultivars (Öz Ayşe and F16) under salt stress (50, 100, 150, $200 \mathrm{mM} \mathrm{NaCl})$ were investigated to determine the hormetic effect of low-dose gamma rays $\left(10,20,30,40 \mathrm{~Gy}{ }^{60} \mathrm{Co}\right)$. The irradiated mature embryos of seeds were regenerated in vitro using embryo cultures. The effects of gamma rays on vegetative growth and amino acid profile under stress and non-stress conditions differed. In terms of vegetative growth, 10 Gy for 'Öz Ayşe' and 20 Gy for 'F16' had hormetic effects and stimulated plant growth under non-stress conditions. Under salt stress conditions, the effect of gamma rays varied according to the severity of the stress. $30 \mathrm{~Gy}$ for 'Öz Ayşe' and 'F16' at 100 $\mathrm{mM}$ salt stress had hormetic effects on vegetative growth. The combined effects of salt stress and gamma rays on the amino acid profile are supported by the vegetative growth results of the plants. Amino acid accumulation occurred at 100 $\mathrm{mM}$ at $30 \mathrm{~Gy}$ for 'Öz Ayşe' and 'F16'. The most accumulated amino acids were glutamic acid (127.63 $\left.\mathrm{mg} \mathrm{kg}^{-1}\right)$, alanine $\left(173.07 \mathrm{mg} \mathrm{kg}^{-1}\right)$, glutamine (188.96 mg kg-1), proline $\left(124.50 \mathrm{mg} \mathrm{kg}^{-1}\right)$, tyrosine (29.23 mg kg-1) in 'Öz Ayşe' and tyrosine $\left(29.88 \mathrm{mg} \mathrm{kg}^{-1}\right)$ in 'F16' under stress. This study shows that low-dose gamma application under moderate stress conditions has a hormetic effect, not under severe salt stress.
\end{abstract}

Key words: Amino acid, common bean, embryo culture, hormesis, low-dose gamma.

\section{INTRODUCTION}

Hormesis is a term used to express the promoting effect of low doses of toxic substances and the suppressive effect of high doses (Calabrese, 2019). Hormetins, or stress sources, are factors that stimulate hormesis. If the stress causes an effect similar to the stimulating effect created by a low dose of a toxin, it is defined as "eustress", namely a beneficial effect, but if it causes irreversible or negative damage, it is defined as "distress", namely harmful stress (Vargas-Hernandez et al., 2017). High doses of ionizing radiation are harmful to plants. However, the concept of high dose differs in plants compared to other organisms. The presence of a cell wall in plants and the totipotency of cells that provide regeneration capability, as well as the presence of a more effective repair system for cellular damage due to the inability of plants to move, result in increased resistance to ionizing radiation (Fornalski et al., 2012). Gamma doses lower than $1 \mathrm{kGy}$ are considered low doses (Iglesias-Andreu et al., 2012), and low-dose gamma application has shown that germination rate and seedling growth in cucumber and okra (Jaipo et al., 2019), germination, fruit number and total yield in tomato (Wiendl et al., 2013), germination index, root and hypocotyl length and photosynthetic pigment content in lettuce (Marcu et al., 2013) are positively affected. Wang et al. (2017) stated that low-dose gamma can affect the expression of genes related to heavy metal transport and abscisic acid metabolism under $\mathrm{Pb} / \mathrm{Cd}$ stress in barley. Saputro et al. (2019) stated that $25 \mathrm{~Gy}$ gamma application positively affected some plant growth parameters under waterlogging stress and caused overexpression of some proteins that are estimated to be 1-aminocyclopropane-1-carboxylate synthase, alcohol-dehydrogenase, and 
superoxide dismutase. Today, research on radiation hormesis is focused on morphological and photosynthetic changes as well as plant nutrient uptake and yield, whereas studies on biochemical changes are limited.

Common beans (Phaseolus vulgaris L.) are an important source of protein, vitamins and minerals as well as an important product for sustainable agriculture. Common beans, which are sensitive to abiotic stress, lose $85 \%$ pod yield per plant when they are exposed to a salinity equivalent to $100 \mathrm{mM} \mathrm{NaCl}$ (De Pascale et al., 1997). Amino acids, which act as growth factors in plants, are the building blocks of proteins. They are important to stimulate plant growth, act as buffers that help maintain the proper $\mathrm{pH}$ in plant cells, are sources of $\mathrm{C}$ and energy, contribute to ammonia removal from the cell because they contain both acidic and basic groups, and facilitate the synthesis of different organic compounds such as enzymes (Ali et al., 2019). Amino acid and protein synthesis are high in growing and differentiating cells, and protein and amino acid breakdown increases during aging. Protein and amino acid degradation can also occur under stress conditions (Hildebrandt et al., 2015).

Approximately one-fifth of the arable land in the world is affected by salt stress and every year 1.5 million hectares of land become unsuitable for agriculture (Hasanuzzaman et al., 2014). Global warming has made this stress even more important. Due to tolerance to salt stress is controlled by multiple genes (Zhang et al., 2013), the transfer of these genes in breeding studies is difficult. Different applications are made to overcome this problem. It is necessary to investigate the hormetic effects of gamma rays, which are used in many different industrial areas other than breeding (Aquino, 2012), and the possibilities of use as a pre-treatment should be investigated. The changes caused by this easy and practicable method in the plant biochemical mechanism are not completely known. In vitro studies are important in terms of creating a model before large-scale field studies, as they can get faster results in smaller areas. The use of in vitro culture methods provides many advantages in determining the tolerance of plants not only to salt stress but also to other abiotic and biotic stress conditions. The elimination of the large land need, which is necessary especially in the field conditions, saves time, labor and cost for breeders and researchers. Salt stress tolerance has been successfully carried out in vitro in many economically important plants such as tomato (Zaki and Yokoi, 2016), spinach (Muchate et al., 2019) and potato (Ahmed et al., 2020). This study was aimed to reveal the relationships between low dose gamma rays and changes of amino acid profiles of common beans in non-stress and salt stress conditions depending on the hormetic effect in vitro.

\section{MATERIALS AND METHODS}

\section{Plant material and embryo culture}

It was used two standard common bean (Phaseolus vulgaris L.) cultivars, Öz Ayşe and F16. The source of gamma radiation was cobalt-60 (Ob-Servo Sanguis Co-60, Institute of Isotopes Co., Ltd. [IZOTOP], Budapest, Hungary), with a dose rate $2190 \mathrm{kGy} \mathrm{h}^{-1}$, at the Turkey Atomic Energy Agency. To determine the effect of low-dose gamma irradiation, we irradiated common bean seeds with four different doses (10, 20, 30, $40 \mathrm{~Gy})$. Unirradiated seeds of the cultivars were used as the control group.

The embryo was cultured under in vitro conditions immediately after the seeds were irradiated. The seeds were shaken continuously for $10 \mathrm{~min}$ in a $10 \%$ sodium hypochlorite solution in a sterile cabinet for surface sterilization. Subsequently, they were placed in $70 \%$ ethanol for $5 \mathrm{~min}$ and washed three times with distilled sterile water, followed by placing them in distilled sterile water for $12 \mathrm{~h}$. The next day, embryos were taken from seeds and transferred to Murashige and Skooge medium $\left(\mathrm{MS}_{0}\right)$ without plant growth regulators containing $30 \mathrm{~g} \mathrm{~L}^{-1}$ sucrose and $7 \mathrm{~g} \mathrm{~L}^{-1}$ agar. Plant growth regulators were not added to any of the media used in the experiment. While only $\mathrm{MS}_{0}$ medium was used for the control group, 50, 100, 150 and $200 \mathrm{mM} \mathrm{NaCl}$ was added to the $\mathrm{MS}_{0}$ media for salt stress treatments. Cultures were maintained at $24 \pm 2{ }^{\circ} \mathrm{C}$ and a 16:8 $\mathrm{h}$ photoperiod. The experiment was a randomized block trial design with 10 replicates and 10 embryos per replicate. Each Magenta plant culture box $(6 \times 6 \mathrm{~cm})$ containing 10 embryos was designed as a replicate. Amino acid profiling analyses were performed $21 \mathrm{~d}$ after the start of the experiment on leaf samples taken from randomly selected plants.

\section{Extraction of free amino acids}

Free amino acids (lysine, Lys; histidine, His; arginine, Arg; cysteine, Cys; serine, Ser; glutamic acid, Glu; threonine, Thr; aspartic acid, Asp; proline, Pro; valine, Val; methionine, Met; tyrosine, Tyr; isoleucine, Ile; leucine, Leu; phenylalanine, Phe) in the ground leaf samples were analyzed using an ultra-high performance liquid chromatography tandem mass spectrometry (UHPLC-MS/MS) device according to the method specified by Nimbalkar et al. (2012). Briefly, $0.5 \mathrm{~g}$ 
homogenized sample was extracted with $10 \mathrm{~mL}$ water:methanol (80:20) (v/v) solution containing $0.1 \%(\mathrm{v} / \mathrm{v})$ formic acid. The mixture was vortexed for $5 \mathrm{~min}$ and then centrifuged at $4000 \mathrm{rpm}$ for $15 \mathrm{~min}$ at $4{ }^{\circ} \mathrm{C}$. The upper phase obtained after centrifugation was passed through a $0.2 \mu \mathrm{m}$ polytetrafluoroethylene (PTFE) membrane filter and injected into the UHPLCMS/MS device. Total analysis time was $7 \mathrm{~min}$. Free amino acid profile analysis and determination were performed using a mass spectrometer system (UltiMate 3000 UHPLC, TSQ Fortis, Thermo Scientific, Waltham, Massachusetts, USA). Chromatographic evaluations were made via the Xcalibur software (Thermo Scientific). A Hypersil Gold RP C18 (1.9 $\mu \mathrm{m})$, $50 \times 2.1-\mathrm{mm}$ UHPLC, column was used as analytical column for chromatographic separation.

\section{Statistical analysis}

The data obtained at the end of the study were analyzed using the Minitab 17.0 package program (Minitab, State College, Pennsylvania, USA). ANOVA test was used to determine the variations and means were compared by Tukey test. The evaluation and comparison of the confidence intervals of the mean of the treatment were performed with the Interval Plot (Minitab).

\section{RESULTS}

\section{Effect of low dose gamma and salt stress on vegetative growth}

Shoot length (SL), root length (RL), leaf width (LW), leaf length (LL), shoot fresh weight (SFW), and root fresh weight (RFW) of the two common bean cultivars, together with the statistical analysis results, are presented in Table 1. It was found a significant difference between cultivars in LL, LW, and RFW. Low-dose gamma rays and salt stress, and combinations of low-dose gamma rays and salt stress, had significant effects on all parameters $(\mathrm{p}<0.01)$. In the interactions between variation sources, a Cultivar $(C) \times$ Low dose $(\mathrm{L})$ interaction in all growth properties, except for SL, and a C $\times$ Salt stress (S) interaction for all parameters were significant at the 0.05 and 0.01 levels. The $\mathrm{C} \times \mathrm{L} \times \mathrm{S}$ triple interaction for all properties was significant at the 0.01 level.

The results of the statistical analysis of the vegetative growth of common bean cultivars treated with different lowdose gamma irradiation are shown in Table 2. Hormetic effect doses differed according to genotypes. Gamma doses had nonsignificant effect on the SL of 'Öz Ayşe'. Further, $10 \mathrm{~Gy}$ had a significant effect on RL (98.50 mm), LL (22.11 mm), LW ( $21.70 \mathrm{~mm}$ ), SFW (0.3536 g), and RFW (0.5898 g). In 'F16', the stimulating effect of $20 \mathrm{~Gy}$ on SL ( $80.11 \mathrm{~mm})$, RL (114.6 $\mathrm{mm})$, LL $(26.34 \mathrm{~mm})$, shoot $(0.3480 \mathrm{~g})$, and root $(0.5468 \mathrm{~g})$ fresh weight was significant. There was nonsignificant effect of low-dose gamma rays' application on LW in 'F16'. However, the highest value $(13.66 \mathrm{~mm})$ was measured at $20 \mathrm{~Gy}$.

Table 3 shows the statistical analysis results of the growth parameters of the embryos regenerated in MS media containing different salt concentrations. Vegetative plantlet growth decreased due to the increase in salt concentration. Especially when the salt concentration was increased to $100 \mathrm{mM}$, the decline in the growth was obvious. In 'Öz Ayşe', SL $(59.22 \mathrm{~mm}), \mathrm{RL}(46.06 \mathrm{~mm})$, and RFW $(0.4240 \mathrm{~g})$ obtained at a salt concentration of $50 \mathrm{mM}$ were included in the same statistical group as the control. In addition, SFW $(0.4380 \mathrm{~g})$ was higher than in the control (0.3984). Although there was nonsignificant difference among treatments in terms of LW and RFW in 'F16', the highest numerical value was obtained at a salt concentration of $50 \mathrm{mM}$. Similar to the 'Öz Ayşe', there was nonsignificant difference between the control (38.54 mm) and $50 \mathrm{mM}(36.62 \mathrm{~mm})$ for SL in 'F16'. The significantly highest values in RL and SFW were obtained from regenerated embryos at $50 \mathrm{mM}$. Control plantlets had the highest values in terms of LL $(11.90 \mathrm{~mm})$.

Table 1. Variance analysis result with respect to growth parameters of common bean cultivars.

\begin{tabular}{|c|c|c|c|c|c|c|c|}
\hline Variation sources & df & SL & RL & LL & LW & SFW & RFW \\
\hline Cultivar (C) & 1 & ns & ns & * & $* *$ & ns & $* *$ \\
\hline Low-dose (L) & 4 & $*$ & $* *$ & $* *$ & $*$ & $*$ & * \\
\hline Salt stress (S) & 4 & $* *$ & $* *$ & ** & ** & $* *$ & $* *$ \\
\hline $\mathrm{C} \times \mathrm{L}$ & 4 & ns & * & $* *$ & * & $* *$ & $* *$ \\
\hline $\mathrm{C} \times \mathrm{S}$ & 4 & $*$ & $* *$ & $* *$ & $* *$ & $*$ & $* *$ \\
\hline $\mathrm{C} \times \mathrm{L} \times \mathrm{S}$ & 24 & $* *$ & $* *$ & $* *$ & $* *$ & $* *$ & $* *$ \\
\hline
\end{tabular}

SL: Shoot length; RL: root length; LL: leaf length; LW: leaf width; SFW: shoot fresh weight; RFW: root fresh weight.

Distinct letters in the row indicate significant differences according to Tukey's test $(\mathrm{P} \leq 0.05)$.

*, ** Significant at $\mathrm{p}<0.05$ and $\mathrm{p}<0.01$, respectively. ns: Nonsignificant. 
Table 2. Effects of low-dose gamma on growth parameters in common bean cultivars.

\begin{tabular}{|c|c|c|c|c|c|c|c|}
\hline Cultivar & Treatment & SL & RL & LL & LW & SFW & RFW \\
\hline & Gy & & $\bar{L}$ & & & & \\
\hline \multirow[t]{5}{*}{ Öz Ayşe } & Control & 69.73 & 61.94ab & $22.22 \mathrm{a}$ & $18.68 \mathrm{ab}$ & $0.3984 a$ & $0.5704 \mathrm{a}$ \\
\hline & 10 & 87.65 & $98.50 \mathrm{a}$ & $22.11 \mathrm{a}$ & $21.70 \mathrm{a}$ & $0.3536 a$ & $0.5898 \mathrm{a}$ \\
\hline & 20 & 55.76 & $94.84 \mathrm{a}$ & $10.66 \mathrm{~b}$ & $15.74 \mathrm{ab}$ & $0.2766 a b$ & $0.4844 \mathrm{ab}$ \\
\hline & 30 & 73.98 & 63.74ab & $13.05 \mathrm{~b}$ & $21.12 \mathrm{a}$ & $0.2218 b$ & $0.1803 b c$ \\
\hline & 40 & 51.74 & $45.82 \mathrm{~b}$ & $8.78 \mathrm{~b}$ & $6.82 \mathrm{~b}$ & $0.1985 b$ & $0.1482 \mathrm{c}$ \\
\hline \multirow[t]{6}{*}{ F16 } & & SL & RL & LL & LW & SFW & RFW \\
\hline & Control & $38.54 \mathrm{c}$ & $19.70 \mathrm{~b}$ & $8.06 \mathrm{~b}$ & 8.52 & $0.1382 b$ & $0.0687 \mathrm{~b}$ \\
\hline & 10 & $53.68 \mathrm{bc}$ & $43.88 \mathrm{~b}$ & $8.35 b$ & 8.90 & $0.2466 \mathrm{ab}$ & $0.0560 \mathrm{~b}$ \\
\hline & 20 & $80.11 \mathrm{a}$ & $114.60 \mathrm{a}$ & $26.34 \mathrm{a}$ & 13.66 & $0.3480 \mathrm{a}$ & $0.5468 \mathrm{a}$ \\
\hline & 30 & $62.17 \mathrm{ab}$ & $44.88 \mathrm{~b}$ & $13.92 \mathrm{~b}$ & 11.78 & $0.2332 \mathrm{ab}$ & $0.1264 \mathrm{~b}$ \\
\hline & 40 & $40.36 \mathrm{bc}$ & $32.17 \mathrm{~b}$ & $10.35 b$ & 10.19 & $0.0994 \mathrm{~b}$ & $0.0575 b$ \\
\hline
\end{tabular}

SL: Shoot length; RL: root length; LL: leaf length; LW: leaf width; SFW: shoot fresh weight; RFW: root fresh weight.

Distinct letters in the row indicate significant differences according to Tukey's test $(\mathrm{P} \leq 0.05)$.

Table 3. Effects of salt stress levels on growth parameters in common bean cultivars.

\begin{tabular}{|c|c|c|c|c|c|c|c|}
\hline Cultivar & Treatment & SL & RL & LL & LW & SFW & RFW \\
\hline & $\mathrm{mM}$ & & $\mathrm{mm}$ & & & $\longrightarrow$ & \\
\hline \multirow[t]{5}{*}{ Öz Ayşe } & Control & $69.73 a$ & 61.94ab & $22.22 \mathrm{a}$ & $18.68 \mathrm{ab}$ & $0.3984 \mathrm{a}$ & $0.5704 \mathrm{a}$ \\
\hline & 50 & $59.22 \mathrm{a}$ & $49.06 \mathrm{a}$ & $11.14 \mathrm{~b}$ & $9.02 \mathrm{~b}$ & $0.4380 \mathrm{a}$ & $0.4240 \mathrm{a}$ \\
\hline & 100 & $25.08 \mathrm{~b}$ & $26.32 b$ & $10.82 b$ & $7.90 \mathrm{~b}$ & $0.3050 \mathrm{bc}$ & $0.0841 \mathrm{~b}$ \\
\hline & 150 & $21.86 \mathrm{bc}$ & $20.48 b c$ & $6.78 \mathrm{c}$ & $6.56 \mathrm{~b}$ & $0.2282 \mathrm{c}$ & $0.1776 \mathrm{~b}$ \\
\hline & 200 & $5.53 \mathrm{c}$ & $6.82 \mathrm{c}$ & $3.57 \mathrm{~d}$ & $4.88 \mathrm{~b}$ & $0.1071 \mathrm{~d}$ & $0.0220 \mathrm{~b}$ \\
\hline \multirow[t]{6}{*}{ F16 } & & SL & RL & LL & LW & SFW & RFW \\
\hline & Control & $38.54 \mathrm{c}$ & $19.70 \mathrm{~b}$ & $8.06 \mathrm{~b}$ & 8.52 & $0.1382 b$ & $0.0687 \mathrm{~b}$ \\
\hline & 50 & $36.62 \mathrm{a}$ & $54.48 \mathrm{a}$ & $11.90 \mathrm{a}$ & 8.70 & $0.4736 \mathrm{a}$ & 0.0869 \\
\hline & 100 & $18.74 \mathrm{~b}$ & $24.68 \mathrm{~b}$ & $8.50 \mathrm{ab}$ & 6.86 & $0.2736 \mathrm{ab}$ & 0.0299 \\
\hline & 150 & $16.46 \mathrm{~b}$ & $13.38 \mathrm{~b}$ & $6.02 \mathrm{~b}$ & 3.48 & $0.1858 b$ & 0.0252 \\
\hline & 200 & 0.00 & 0.00 & 0.00 & 0.00 & 0.0000 & 0.0000 \\
\hline
\end{tabular}

SL: Shoot length; RL: root length; LL: leaf length; LW: leaf width; SFW: shoot fresh weight; RFW: root fresh weight.

Distinct letters in the row indicate significant differences according to Tukey's test $(\mathrm{P} \leq 0.05)$.

The comparative analysis of the effects of applications on vegetative parameters in 'Öz Ayşe' is presented in Figure 1. There was a hormetic effect of $30 \mathrm{~Gy}$ irradiation on LW $(12.72 \mathrm{~mm})$ and SFW $(0.5944 \mathrm{~g})$ under $50 \mathrm{mM}$ salt stress. In addition, the hormetic effect of $30 \mathrm{~Gy}$ irradiation on SL (42.46 mm), RL (36.88), LW (9.50 mm), shoot (0.3882 g), and RFWs $(0.2522 \mathrm{~g})$ was determined at $100 \mathrm{mM}$ salt concentration, where salt stress had a high negative effect on vegetative growth. Low-dose gamma rays applied for any of the parameters at 150 and $200 \mathrm{mM}$ salt stress had no hormetic effect.

The statistical analysis results of the effects of the applications on the vegetative development of the 'F16' are presented as an interval plot (Figure 2). Although there was germination under $200 \mathrm{mM}$ salt stress, no surviving plant could be obtained. Similarly, the embryos of seeds irradiated with $40 \mathrm{~Gy}$ did not survive in medium with a salt concentration of $150 \mathrm{mM}$. In terms of SL $(46.16 \mathrm{~mm})$, the embryos of seeds treated with low doses of 40 Gy showed a hormetic effect at a salt concentration of $50 \mathrm{mM}$. Similarly, it was determined a stimulating effect of 40 Gy on LW $(9.06 \mathrm{~mm})$ and RFW $(0.2122 \mathrm{~g})$. An irradiation dose of $30 \mathrm{~Gy}$ at salt stress of $100 \mathrm{mM}$ had a hormetic effect on all parameters. When a salt concentration of $150 \mathrm{mM}$ was applied, the effect of the 20 Gy irradiation dose was significant in terms of SL (28.30 $\mathrm{mm})$ and RL $(14.70 \mathrm{~mm})$. All applications were in the same statistical group in respect to LL. The dose of $10 \mathrm{~Gy}$ had a prominent effect on LW $(7.08 \mathrm{~mm})$. All applications on SFW gave better results than $150 \mathrm{mM}$ application and were included in a separate group. A hormetic effect of $10(0.0433 \mathrm{~g})$ and $30 \mathrm{~Gy}(0.0401 \mathrm{~g})$ on RFW was determined. 
Figure 1. Interval plots of the effects of low-dose gamma rays and salt stress on the vegetative growth of common bean 'Öz Ayşe' (mean $\pm 95 \%$ confidence intervals).
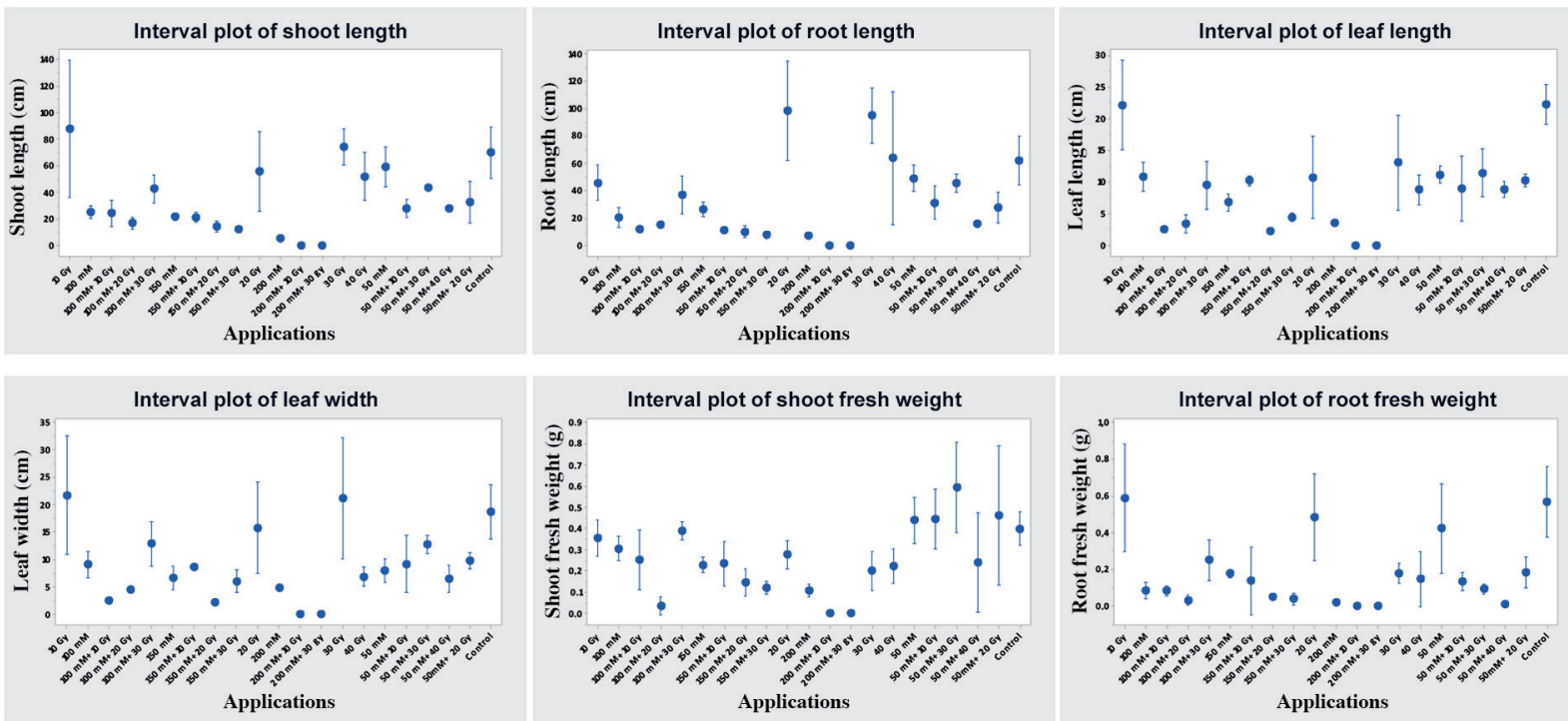

Figure 2. Interval plots of the effects of low-dose gamma and salt stress on the vegetative growth of common bean 'F16' (mean $\pm 95 \%$ confidence intervals).
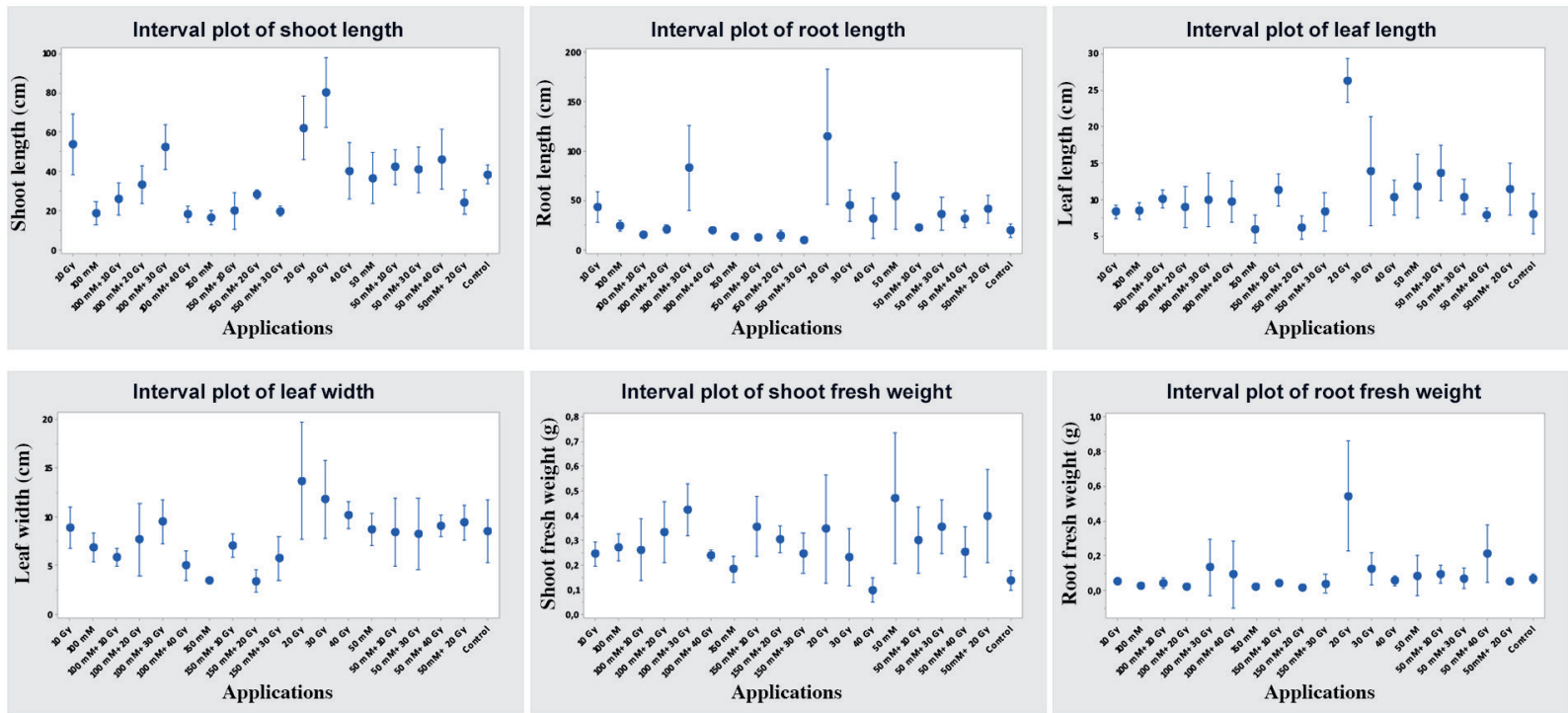

\section{Free amino acid profiling}

Chromatograms and calibration curves of some free amino acids are presented in Figure 3 . The correlation coefficients $\left(\mathrm{R}^{2}\right)$ of linear regression analysis from calibration curves were between 0.9929 and 0.9999 . The limits of detection (LOD) and limits of quantification (LOQ) for sensitivity were evaluated in the ranges of 4.4-7.6 and 14.6-25.5 $\mathrm{mg} \mathrm{kg}^{-1}$, respectively. The relative standard deviations (\% RSD) of the peak corrected areas obtained by injecting amino acid standard solutions six times in a row were determined; the RSD values corresponding to precision were between $2.3 \%$ and $4.1 \%$. The accuracy of the method was determined by adding standard amino acids to the leaf sample whose amino acid analysis was performed and evaluating the recovery. The recovery values varied between $87.2 \%$ and $96.3 \%$. 
Figure 3. Chromatograms and calibration curves of some free amino acids.

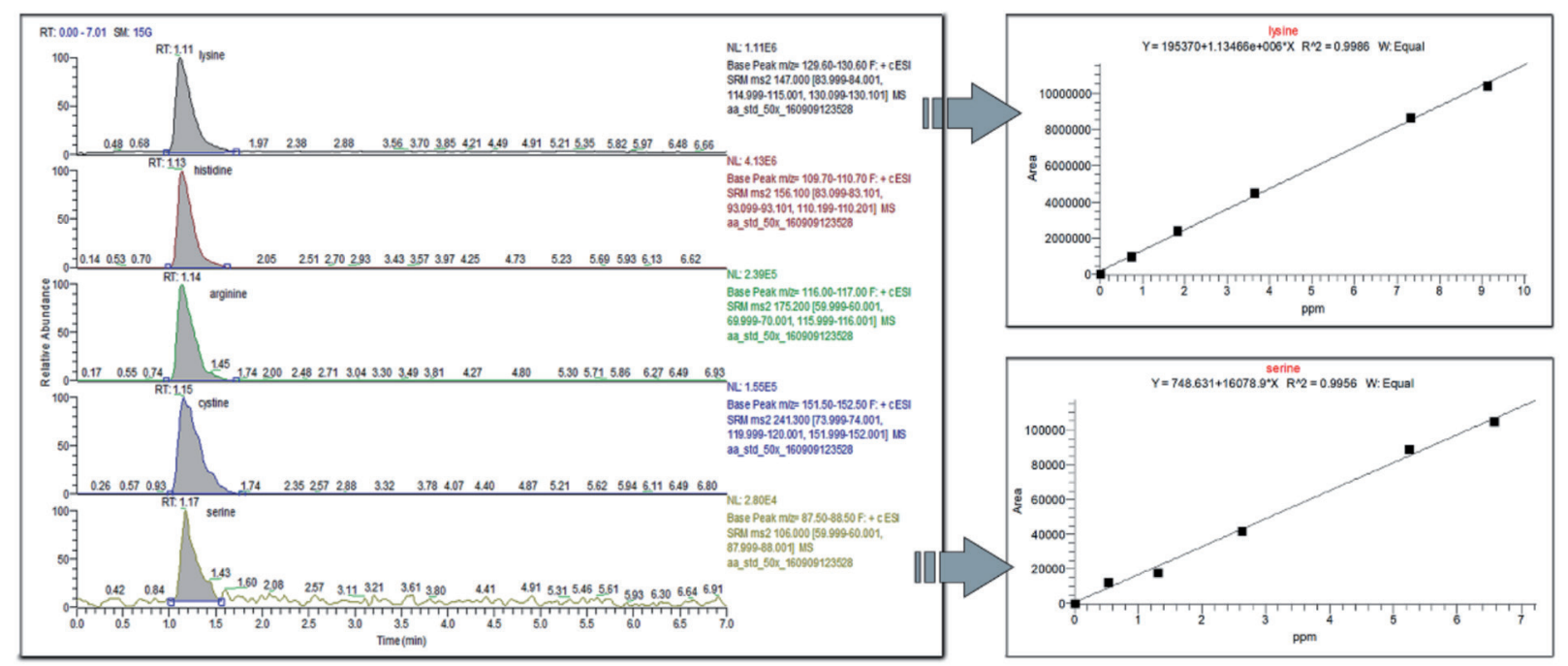

The ANOVA results regarding the amino acid profiles of two common bean cultivars are shown in Table 4. As a source of variation, the cultivars had a significant effect at the level of 0.05 on Thr, Glu, Ala, Pro, Tyr and total free amino acid (TFAA), while on Gln and Val, the effect was significant at 0.01. The effect of low salt stress on Lys, Asp, Ser, Glu, Ala, Gln, Val and TFAA was significant at 0.05, while salt stress had a significant effect on all amino acid contents at 0.01 . The interaction Cultivar $\times$ Low-dose gamma irradiation was significant at 0.05 for Pro and Leu, while for all other amino acids, it was significant at 0.01 . The interaction Variety $\times$ Salt was significant at 0.05 only for Met. For the other amino acids, 0.01 was the significance level. The results of triple interaction Cultivar $\times$ Low-dose gamma rays $\times$ Salt stress revealed that there was a triple interaction at 0.01 for all amino acids.

The amino acid profiles of the common bean cultivars differed according to the gamma ray doses applied (Table 5). For Lys, (43.94 mg kg-1), Cys (14.31 mg kg-1), His (37.04 mg kg-1), Arg (37.46 mg kg-1), Asp (147.55 mg kg-1), Ser (70.08 mg kg-1), Thr (47.25 mg kg-1), Glu (127.29 mg kg-1), Ala (183.25 mg kg-1), Gln (178.82 $\left.\mathrm{mg} \mathrm{kg}^{-1}\right)$, Pro (124.32 mg kg-1), Val (69.01 mg kg $\mathrm{mg}^{-1}$, Ile (69.51 $\left.\mathrm{mg} \mathrm{kg}^{-1}\right)$, Leu (57.28 $\left.\mathrm{mg} \mathrm{kg}^{-1}\right)$, Phe (88.41 $\left.\mathrm{mg} \mathrm{kg}^{-1}\right)$, and TFAA (1307.33 mg kg-1), the levels were highest in the plants obtained from embryos of $40 \mathrm{~Gy}$ irradiated 'Öz Ayşe' seeds. Only the levels of Met $\left(8.9 \mathrm{mg} \mathrm{kg}{ }^{-1}\right)$ and Tyr (23.30 $\left.\mathrm{mg} \mathrm{kg}^{-1}\right)$ increased at $20 \mathrm{~Gy}$ in 'F16', while Met $\left(9.41 \mathrm{mg} \mathrm{kg}^{-1}\right)$ and Tyr $\left(27.29 \mathrm{mg} \mathrm{kg}^{-1}\right)$ were highest at 40 Gy, generally, the amino acid values were increased at $10 \mathrm{~Gy}$. However, Arg (40.70 mg kg-1), Asp (146.26 mg kg-1), Ala (166.24 $\left.\mathrm{mg} \mathrm{kg}^{-1}\right)$, Pro $\left(118.86 \mathrm{mg} \mathrm{kg}^{-1}\right)$ and Val $\left(64.02 \mathrm{mg} \mathrm{kg}^{-1}\right)$ reached the highest values at $20 \mathrm{~Gy}$. Comparing the two cultivars, the amino acid values were generally higher for ' $\mathrm{F} 16$ '.

Table 4. ANOVA result with respect to the amino acid profile of common bean cultivars.

\begin{tabular}{|c|c|c|c|c|c|c|c|c|c|c|}
\hline Variation sources & df & Lys & Cys & His & Arg & Asp & Ser & Thr & Glu & Ala \\
\hline C (Cultivar) & 1 & ns & ns & ns & ns & ns & ns & $*$ & $*$ & $*$ \\
\hline L (Low-dose) & 4 & $*$ & ns & ns & ns & $*$ & $*$ & ns & $*$ & $*$ \\
\hline S (Salt stress) & 4 & $* *$ & $* *$ & $* *$ & $* *$ & $* *$ & $* *$ & $* *$ & $* *$ & $* *$ \\
\hline $\mathrm{C} \times \mathrm{L}$ & 4 & $* *$ & $* *$ & $* *$ & $* *$ & $* *$ & $* *$ & $* *$ & $* *$ & $* *$ \\
\hline $\mathrm{C} \times \mathrm{S}$ & 4 & $* *$ & $* *$ & $* *$ & $* *$ & $* *$ & $* *$ & $* *$ & $* *$ & $* *$ \\
\hline \multirow[t]{2}{*}{$\mathrm{C} \times \mathrm{L} \times \mathrm{S}$} & 24 & $* *$ & $* *$ & $* *$ & $* *$ & $* *$ & $* *$ & $* *$ & $* *$ & $* *$ \\
\hline & & Gln & Pro & Val & Met & Tyr & Ile & Leu & Phe & TFAA \\
\hline $\mathrm{C}$ & 1 & $* *$ & $*$ & $* *$ & ns & $*$ & ns & ns & ns & $*$ \\
\hline $\mathrm{L}$ & 4 & $*$ & ns & $*$ & ns & ns & $\mathrm{ns}$ & ns & ns & $*$ \\
\hline $\mathrm{S}$ & 4 & $* *$ & $* *$ & $* *$ & $* *$ & $* *$ & $* *$ & $* *$ & $* *$ & $* *$ \\
\hline $\mathrm{C} \times \mathrm{L}$ & 4 & $* *$ & $*$ & $* *$ & $* *$ & $* *$ & $* *$ & $*$ & $* *$ & $* *$ \\
\hline $\mathrm{C} \times \mathrm{S}$ & 4 & $* *$ & $* *$ & $* *$ & $*$ & $* *$ & $* *$ & $* *$ & $* *$ & $* *$ \\
\hline $\mathrm{C} \times \mathrm{L} \times \mathrm{S}$ & 24 & $* *$ & $* *$ & $* *$ & $* *$ & $* *$ & $* *$ & $* *$ & $* *$ & $* *$ \\
\hline
\end{tabular}

Ala: Alanine; Arg: arginine; Asp: aspartic acid; Cys: cysteine; Glu: glutamic acid; His: histidine; Ile: isoleucine; Leu: leucine; Lys: lysine; Met: methionine; Phe: phenylalanine; Pro: proline; Ser: serine; Thr: threonine; Tyr: tyrosine; Val: valine; TFAA: total free amino acid.

$*$,**Significant at $\mathrm{p}<0.05$ and $\mathrm{p}<0.01$, respectively. ns: Nonsignificant. 
Table 5. Effects of low dose gamma rays applications on amino acid profile of common bean cultivars.

\begin{tabular}{|c|c|c|c|c|c|c|c|c|c|c|}
\hline Cultivar & Dose & Lys & Cys & His & Arg & Asp & Ser & Thr & Glu & Ala \\
\hline \multirow{12}{*}{ Öz Ayşe } & Gy & & & & & $\mathrm{mg} \mathrm{kg}^{-1}$ & & & & \\
\hline & $\mathrm{Cnt}$ & $32.63 b$ & $12.05 \mathrm{a}$ & $29.44 \mathrm{c}$ & $33.81 \mathrm{~b}$ & $111.39 \mathrm{c}$ & $49.25 \mathrm{c}$ & $35.76 \mathrm{c}$ & $93.08 \mathrm{c}$ & $126.56 \mathrm{c}$ \\
\hline & 10 & $41.54 \mathrm{a}$ & $13.80 \mathrm{a}$ & $33.14 \mathrm{~b}$ & $36.78 \mathrm{a}$ & $132.28 b$ & $62.70 \mathrm{~b}$ & $43.99 \mathrm{~b}$ & $118.51 b$ & $161.13 b$ \\
\hline & 20 & $30.43 b c$ & $6.48 \mathrm{~b}$ & $21.34 \mathrm{~d}$ & $24.86 \mathrm{c}$ & $103.69 d$ & $47.70 \mathrm{c}$ & $21.24 \mathrm{~d}$ & $82.72 d$ & $110.13 \mathrm{~d}$ \\
\hline & 30 & $28.22 \mathrm{c}$ & $6.09 \mathrm{~b}$ & $20.86 \mathrm{~d}$ & $24.30 \mathrm{c}$ & $99.56 \mathrm{e}$ & $44.24 \mathrm{~d}$ & $20.04 \mathrm{~d}$ & $76.72 \mathrm{e}$ & $102.14 \mathrm{e}$ \\
\hline & 40 & $43.94 \mathrm{a}$ & $14.31 \mathrm{a}$ & $37.04 \mathrm{a}$ & $37.46 \mathrm{a}$ & $147.55 \mathrm{a}$ & $70.08 \mathrm{a}$ & $47.25 \mathrm{a}$ & $127.293 \mathrm{a}$ & $183.25 \mathrm{a}$ \\
\hline & & Gln & Pro & Val & Met & Tyr & Ile & Leu & Phe & TFAA \\
\hline & $\mathrm{Cnt}$ & $133.06 \mathrm{c}$ & $90.67 \mathrm{c}$ & $49.61 \mathrm{c}$ & $3.85 \mathrm{c}$ & $4.33 b$ & $50.69 \mathrm{c}$ & $42.62 \mathrm{c}$ & $62.25 \mathrm{c}$ & $961.15 \mathrm{c}$ \\
\hline & 10 & $169.41 \mathrm{~b}$ & $115.44 \mathrm{~b}$ & $63.16 \mathrm{~b}$ & $4.90 \mathrm{bc}$ & $5.51 \mathrm{~b}$ & $64.54 \mathrm{~b}$ & $54.27 \mathrm{~b}$ & $79.26 \mathrm{~b}$ & $1200.46 \mathrm{~b}$ \\
\hline & 20 & $125.11 \mathrm{~d}$ & $75.23 \mathrm{~d}$ & $49.03 c$ & $8.09 \mathrm{a}$ & $23.30 \mathrm{a}$ & $40.33 \mathrm{~d}$ & $37.07 \mathrm{~d}$ & $55.43 \mathrm{~d}$ & $862.25 \mathrm{~d}$ \\
\hline & 30 & $116.03 \mathrm{e}$ & $69.77 \mathrm{e}$ & $45.47 \mathrm{~d}$ & $7.50 \mathrm{ab}$ & $21.61 \mathrm{a}$ & $37.40 \mathrm{e}$ & $34.38 \mathrm{e}$ & $51.40 \mathrm{e}$ & $805.81 \mathrm{e}$ \\
\hline & 40 & $178.82 \mathrm{a}$ & $124.32 \mathrm{a}$ & $69.01 \mathrm{a}$ & $5.57 \mathrm{abc}$ & $6.16 \mathrm{~b}$ & $69.51 \mathrm{a}$ & $57.28 \mathrm{a}$ & $88.41 \mathrm{a}$ & $1307.33 \mathrm{a}$ \\
\hline \multirow[t]{12}{*}{ F16 } & & Lys & Cys & His & $\operatorname{Arg}$ & Asp & Ser & Thr & Glu & Ala \\
\hline & $\mathrm{Cnt}$ & $48.95 b$ & $13.80 \mathrm{ab}$ & $38.71 \mathrm{a}$ & $34.01 \mathrm{c}$ & $142.82 \mathrm{~b}$ & $73.80 \mathrm{~b}$ & $42.70 \mathrm{bc}$ & $122.18 \mathrm{~b}$ & $159.66 \mathrm{~b}$ \\
\hline & 10 & $51.76 \mathrm{a}$ & $15.86 \mathrm{a}$ & $40.83 \mathrm{a}$ & $36.50 \mathrm{~b}$ & $144.10 \mathrm{ab}$ & $76.36 \mathrm{a}$ & $46.33 a$ & $126.03 \mathrm{a}$ & $167.76 \mathrm{a}$ \\
\hline & 20 & $41.97 \mathrm{c}$ & $13.69 \mathrm{ab}$ & $35.29 \mathrm{~b}$ & $40.70 \mathrm{a}$ & $146.26 \mathrm{a}$ & $61.22 \mathrm{c}$ & $43.69 \mathrm{ab}$ & $119.52 \mathrm{c}$ & $166.24 \mathrm{a}$ \\
\hline & 30 & $35.81 \mathrm{~d}$ & $12.32 \mathrm{~b}$ & $31.31 \mathrm{c}$ & $32.83 \mathrm{c}$ & $118.26 \mathrm{~d}$ & $55.04 \mathrm{~d}$ & $39.40 \mathrm{c}$ & $98.69 \mathrm{~d}$ & $134.01 \mathrm{c}$ \\
\hline & 40 & $35.11 \mathrm{~d}$ & $7.73 \mathrm{c}$ & $24.52 d$ & $28.61 \mathrm{~d}$ & $121.82 \mathrm{c}$ & $53.31 \mathrm{~d}$ & $23.99 d$ & $96.71 \mathrm{~d}$ & $124.39 \mathrm{~d}$ \\
\hline & & Gln & Pro & Val & Met & Tyr & Ile & Leu & Phe & TFAA \\
\hline & Cnt & $179.41 \mathrm{a}$ & $105.92 \mathrm{c}$ & $61.67 \mathrm{a}$ & $5.87 \mathrm{~b}$ & $6.50 \mathrm{bc}$ & $77.23 \mathrm{a}$ & $63.88 \mathrm{a}$ & $90.18 b$ & $1267.36 b$ \\
\hline & 10 & $183.00 \mathrm{a}$ & $112.10 \mathrm{~b}$ & $62.93 \mathrm{a}$ & $6.06 \mathrm{~b}$ & $6.93 b$ & $83.13 \mathrm{a}$ & $66.46 \mathrm{a}$ & $94.86 \mathrm{a}$ & $1321.07 \mathrm{a}$ \\
\hline & 20 & $162.17 b$ & $118.86 \mathrm{a}$ & $64.02 \mathrm{a}$ & $4.97 \mathrm{~b}$ & $5.78 \mathrm{bc}$ & $62.95 \mathrm{~b}$ & $54.02 \mathrm{~b}$ & $75.87 \mathrm{c}$ & $1217.29 \mathrm{c}$ \\
\hline & 30 & $138.75 d$ & $91.05 \mathrm{~d}$ & $50.72 \mathrm{c}$ & $4.31 \mathrm{~b}$ & $4.51 \mathrm{c}$ & $50.90 \mathrm{c}$ & $45.14 \mathrm{c}$ & $68.32 \mathrm{~d}$ & $1009.72 \mathrm{~d}$ \\
\hline & 40 & $144.17 \mathrm{c}$ & $88.39 \mathrm{e}$ & $57.50 \mathrm{~b}$ & $9.31 \mathrm{a}$ & $27.29 \mathrm{a}$ & $46.41 \mathrm{c}$ & $44.16 \mathrm{c}$ & $62.66 \mathrm{e}$ & $997.90 \mathrm{e}$ \\
\hline
\end{tabular}

Cnt: Control; Ala: alanine; Arg: arginine; Asp: aspartic acid; Cys: cysteine; Glu: glutamic acid; His: histidine; Ile: isoleucine; Leu: leucine; Lys: lysine; Met: methionine; Phe: phenylalanine; Pro: proline; Ser: serine; Thr: threonine; Tyr: tyrosine; Val: valine; TFAA: total free amino acid.

Distinct letters in the row indicate significant differences according to Tukey's test $(\mathrm{P} \leq 0.05)$.

The responses of the cultivars to different salt stress levels in terms of amino acid amounts differed (Table 6). In 'Öz Ayşe', the highest values were generally detected in plants obtained from embryos cultured at a salt concentration of 100 $\mathrm{mM}$, where the physiological effects of salt stress could be observed. In contrast the levels of Met $\left(8.84 \mathrm{mg} \mathrm{kg}^{-1}\right)$ and Tyr $\left(25.06 \mathrm{mg} \mathrm{kg}^{-1}\right)$ increased at $150 \mathrm{mM}$. A more complex situation was observed for ' $\mathrm{F} 16$ '. While the highest amounts of Cys $\left(11.41 \mathrm{mg} \mathrm{kg}^{-1}\right)$, His $\left(30.52 \mathrm{mg} \mathrm{kg}^{-1}\right)$, Arg $\left(34.53 \mathrm{mg} \mathrm{kg}^{-1}\right)$, Thr (37.02 $\left.\mathrm{mg} \mathrm{kg}^{-1}\right)$, Glu $\left(96.37 \mathrm{mg} \mathrm{kg}^{-1}\right)$, Pro (96.06 mg kg $\left.\mathrm{g}^{-1}\right)$, Ile (48.89 $\left.\mathrm{mg} \mathrm{kg}^{-1}\right)$, and Leu (45.08 $\mathrm{mg} \mathrm{kg}^{-1}$ ) were detected at $100 \mathrm{mM}$; Lys (36.90 $\left.\mathrm{mg} \mathrm{kg}^{-1}\right)$, Asp (123.12 $\left.\mathrm{mg} \mathrm{kg}^{-1}\right)$, Ser (56.55 $\left.\mathrm{mg} \mathrm{kg}{ }^{-1}\right)$, Ala (128.36 mg kg-1), Gln (143.30 mg kg$\left.{ }^{-1}\right)$, Val (56.27 $\left.\mathrm{mg} \mathrm{kg}^{-1}\right)$, Met (9.10 $\left.\mathrm{mg} \mathrm{kg}^{-1}\right)$, Tyr $\left(27.77 \mathrm{mg} \mathrm{kg}^{-1}\right)$, Phe $\left(67.22 \mathrm{mg} \mathrm{kg}^{-1}\right)$, and TFAA $\left(999.00 \mathrm{mg} \mathrm{kg}^{-1}\right)$ reached the highest values at $150 \mathrm{mM}$. Unlike for low gamma rays doses, when each stress level was examined separately, the amino acid amounts of 'Oz Ayşe' were generally higher.

The interval plot in Figure 4 allows the comparison of all applications and combinations of these applications. We observed differences in the amino acid values of 'Öz Ayşe' depending on low-dose gamma rays applications and salt stress. Generally, amino acid accumulation occurred at $30 \mathrm{~Gy}$ at 50 and $100 \mathrm{mM} \mathrm{NaCl}$. It cannot be said that a single irradiation dose stands out at $150 \mathrm{mM}$.

Low-dose gamma irradiation and salt stress applications caused differences in the plant amino acid profile in 'F16' (Figure 5). Significantly highest values were generally obtained for control and $30 \mathrm{~Gy}$ gamma irradiation. In general, amino acid accumulation occurred at $40 \mathrm{~Gy}$ at $50 \mathrm{mM}$ and $30 \mathrm{~Gy}$ at $100 \mathrm{mM}$. Similar to 'Öz Ayşe' at $150 \mathrm{mM}$, it was determined that there was no relationship between the increase in amino acid amount and gamma rays dose. On the other hand, there was nonsignificant difference in Thr amounts among the applications $(\mathrm{p}<0.05)$. 
Table 6. Effects of salt stress levels on amino acid profile of common bean cultivars.

\begin{tabular}{|c|c|c|c|c|c|c|c|c|c|c|}
\hline Cultivar & $\mathrm{NaCl}$ & Lys & Cystine & His & Arg & Asp & Ser & Thr & Glu & Ala \\
\hline & $\mathrm{mM}$ & & & & & $\mathrm{mg} \mathrm{kg}^{-1}$ & & & & \\
\hline \multirow[t]{11}{*}{ Öz Ayşe } & Cnt & $32.63 \mathrm{c}$ & $12.05 \mathrm{~b}$ & $29.44 \mathrm{c}$ & $33.81 \mathrm{~b}$ & $111.39 \mathrm{c}$ & $49.25 \mathrm{c}$ & $35.76 \mathrm{c}$ & $93.08 \mathrm{c}$ & $126.56 \mathrm{c}$ \\
\hline & 50 & $36.32 \mathrm{~b}$ & $12.28 \mathrm{~b}$ & $32.22 \mathrm{~b}$ & $38.37 \mathrm{a}$ & $126.38 \mathrm{~b}$ & $53.89 \mathrm{~b}$ & $39.97 b$ & $96.53 b$ & $135.93 \mathrm{~b}$ \\
\hline & 100 & $45.46 \mathrm{a}$ & $15.13 \mathrm{a}$ & $37.69 \mathrm{a}$ & $38.86 \mathrm{a}$ & $142.66 \mathrm{a}$ & $65.02 \mathrm{a}$ & $48.06 \mathrm{a}$ & $129.90 \mathrm{a}$ & $173.29 \mathrm{a}$ \\
\hline & 150 & $34.61 b c$ & $7.09 \mathrm{c}$ & $24.21 \mathrm{~d}$ & $28.21 \mathrm{c}$ & $111.66 \mathrm{c}$ & $49.50 \mathrm{c}$ & $22.82 \mathrm{e}$ & $90.67 \mathrm{c}$ & $118.77 \mathrm{~d}$ \\
\hline & 200 & $28.97 \mathrm{~d}$ & $9.98 \mathrm{~b}$ & $25.31 d$ & $24.72 d$ & $125.72 d$ & $42.28 \mathrm{~d}$ & $32.36 \mathrm{~d}$ & $84.26 \mathrm{~d}$ & $112.52 \mathrm{e}$ \\
\hline & & Gln & Pro & Val & Met & Tyr & Ile & Leu & Phe & TFAA \\
\hline & Cnt & $133.06 \mathrm{~d}$ & $90.67 \mathrm{c}$ & $49.61 \mathrm{c}$ & $3.85 \mathrm{~b}$ & $4.33 b$ & $50.69 \mathrm{c}$ & $42.62 b$ & $62.25 \mathrm{c}$ & $961.15 \mathrm{c}$ \\
\hline & 50 & $148.42 b$ & $101.14 \mathrm{~b}$ & $55.23 \mathrm{~b}$ & $4.15 b$ & $4.41 \mathrm{~b}$ & $55.39 \mathrm{~b}$ & $44.99 \mathrm{~b}$ & $65.71 b$ & $1051.41 \mathrm{~b}$ \\
\hline & 100 & $181.96 \mathrm{a}$ & $126.76 \mathrm{a}$ & $64.33 \mathrm{a}$ & $5.37 \mathrm{~b}$ & $6.14 b$ & $69.41 \mathrm{a}$ & $58.37 \mathrm{a}$ & $86.74 a$ & $1295.24 \mathrm{a}$ \\
\hline & 150 & $139.83 c$ & $82.47 d$ & $55.63 \mathrm{~b}$ & $8.84 \mathrm{a}$ & $25.06 \mathrm{a}$ & $44.14 \mathrm{~d}$ & $37.76 \mathrm{c}$ & $62.89 \mathrm{c}$ & $944.22 \mathrm{c}$ \\
\hline & 200 & $120.62 \mathrm{e}$ & $77.92 \mathrm{e}$ & $44.98 \mathrm{~d}$ & $3.49 \mathrm{~b}$ & $3.62 b$ & $44.30 \mathrm{~d}$ & $36.39 \mathrm{c}$ & $55.26 \mathrm{~d}$ & $889.18 \mathrm{c}$ \\
\hline \multirow[t]{12}{*}{ F16 } & & Lys & Cys & His & Arg & Asp & Ser & Thr & Glu & Ala \\
\hline & Cnt & $48.95 \mathrm{a}$ & $13.80 \mathrm{a}$ & $38.71 \mathrm{a}$ & $34.53 a$ & $142.82 \mathrm{a}$ & $73.80 \mathrm{a}$ & $42.70 \mathrm{a}$ & $122.18 \mathrm{a}$ & $159.66 \mathrm{a}$ \\
\hline & 50 & $28.56 \mathrm{~d}$ & $9.97 \mathrm{bc}$ & $25.27 \mathrm{c}$ & $27.14 b$ & $90.44 d$ & $43.03 \mathrm{~d}$ & $30.12 \mathrm{c}$ & $82.93 \mathrm{c}$ & $110.41 \mathrm{~d}$ \\
\hline & 100 & $33.16 \mathrm{c}$ & $11.41 \mathrm{ab}$ & $30.52 b$ & $34.53 \mathrm{a}$ & $111.72 \mathrm{c}$ & $50.29 \mathrm{c}$ & $37.02 \mathrm{~b}$ & $96.37 \mathrm{~b}$ & $122.07 \mathrm{c}$ \\
\hline & 150 & $36.90 \mathrm{~b}$ & $7.56 \mathrm{c}$ & $24.93 \mathrm{c}$ & $27.98 b$ & $123.12 b$ & $56.55 \mathrm{~b}$ & $23.90 \mathrm{~d}$ & $94.94 \mathrm{~b}$ & $128.36 \mathrm{~b}$ \\
\hline & 200 & 0.00 & 0.00 & 0.00 & 0.00 & 0.00 & 0.00 & 0.00 & 0.00 & 0.00 \\
\hline & & Gln & Pro & Val & Met & Tyr & Ile & Leu & Phe & TFAA \\
\hline & Cnt & $179.41 \mathrm{a}$ & $105.92 \mathrm{a}$ & $61.67 \mathrm{a}$ & $5.87 \mathrm{~b}$ & $6.50 \mathrm{~b}$ & $77.23 \mathrm{a}$ & $63.88 \mathrm{a}$ & $90.18 \mathrm{a}$ & $1267.36 \mathrm{a}$ \\
\hline & 50 & $114.40 \mathrm{~d}$ & $77.84 d$ & $41.74 d$ & $3.29 \mathrm{~b}$ & $3.78 \mathrm{c}$ & $44.99 \mathrm{c}$ & $35.24 \mathrm{c}$ & $51.47 \mathrm{~d}$ & $820.71 d$ \\
\hline & 100 & $138.32 \mathrm{c}$ & $94.06 \mathrm{~b}$ & $50.66 \mathrm{c}$ & $4.06 \mathrm{~b}$ & $4.41 \mathrm{bc}$ & $48.89 \mathrm{~b}$ & $45.08 \mathrm{~b}$ & $64.58 \mathrm{c}$ & $977.25 \mathrm{c}$ \\
\hline & 150 & $143.30 \mathrm{~b}$ & $83.15 \mathrm{c}$ & $56.27 \mathrm{~b}$ & $9.10 \mathrm{a}$ & $27.77 \mathrm{a}$ & $44.57 \mathrm{c}$ & $43.32 b$ & $67.22 \mathrm{~b}$ & $999.00 \mathrm{~b}$ \\
\hline & 200 & 0.00 & 0.00 & 0.00 & 0.00 & 0.00 & 0.00 & 0.00 & 0.00 & 0.00 \\
\hline
\end{tabular}

Cnt: Control; Ala: alanine; Arg: arginine; Asp: aspartic acid; Cys: cysteine; Glu: glutamic acid; His: histidine; Ile: isoleucine; Leu: leucine; Lys: lysine; Met: methionine; Phe: phenylalanine; Pro: proline; Ser: serine; Thr: threonine; Tyr: tyrosine; Val: valine; TFAA: total free amino acid. Distinct letters in the row indicate significant differences according to Tukey's test $(\mathrm{P} \leq 0.05)$.

\section{DISCUSSION}

The hormetic effect of low-dose gamma rays was determined based on the comparison of vegetative growth and amino acid profiles of irradiated and non-irradiated common bean seeds under salt stress conditions. Low-dose gamma irradiation and salt stress had significant effects on vegetative growth. The stimulating effect of gamma rays is based on accelerating cell division, positively affecting vegetative growth (Marcu et al., 2013). Root development is an important parameter in salt stress. In 'Öz Ayşe', 10 Gy increased RL. The most effective irradiation dose in 'F16' was 20 Gy; it dramatically increased RL and, considering its effect on RFW, plays a role not only in the elongation of roots but also in the production of organic matter. In a study conducted on cowpea, low-dose gamma rays application increased mitotic activity at the root tips in $\mathrm{M}_{1}$ and $\mathrm{M}_{2}$ plants (Badr et al., 2014). Similarly, low-dose gamma rays application in onion causes an increase in the proportion of dividing cells (Kumar et al., 2011). In addition, 10 Gy gamma irradiation positively affected SL, RFW, SFW and leaf development in 'Öz Ayşe'. Salt stress adversely affected plant growth as expected. In both cultivars, this negative effect was clearly observed at $100 \mathrm{mM}$. The counteractive effect of low-dose gamma rays against this negative influence was determined at $30 \mathrm{~Gy}$ at 50 and $100 \mathrm{mM}$ in 'Öz Ayşe'. In 'F16', $30 \mathrm{~Gy}$ for $100 \mathrm{mM}$ salt stress had hormetic effects. The results obtained were consistent with the findings of studies performed to demonstrate the hormetic effect of lowdose gamma irradiation on apricot (El-Sabagh et al., 2011), lettuce (Marcu et al., 2013), and Arabidopsis (Qi et al., 2014). Zaka et al. (2004) drew attention to point mutations that occur in apical meristem cells caused by low-gamma irradiation at an early stage of plant development. The authors state that the repair mechanism is triggered at high gamma doses, but at low doses, it is transferred without induction of the repair mechanism, and accumulation occurs during successive cell divisions. Low-dose gamma irradiation accelerates cell division, whereas the repair mechanism is not induced; this explains the increase in plant growth. Changes caused by gamma rays in chromosomes vary depending on the dose and variety. 
Figure 4. Interval plot of the effect of $\mathrm{NaCl}$ and low-dose gamma ray applications on the amino acid profile of 'Öz Ayşe' cultivar (mean $\pm 95 \%$ confidence intervals).
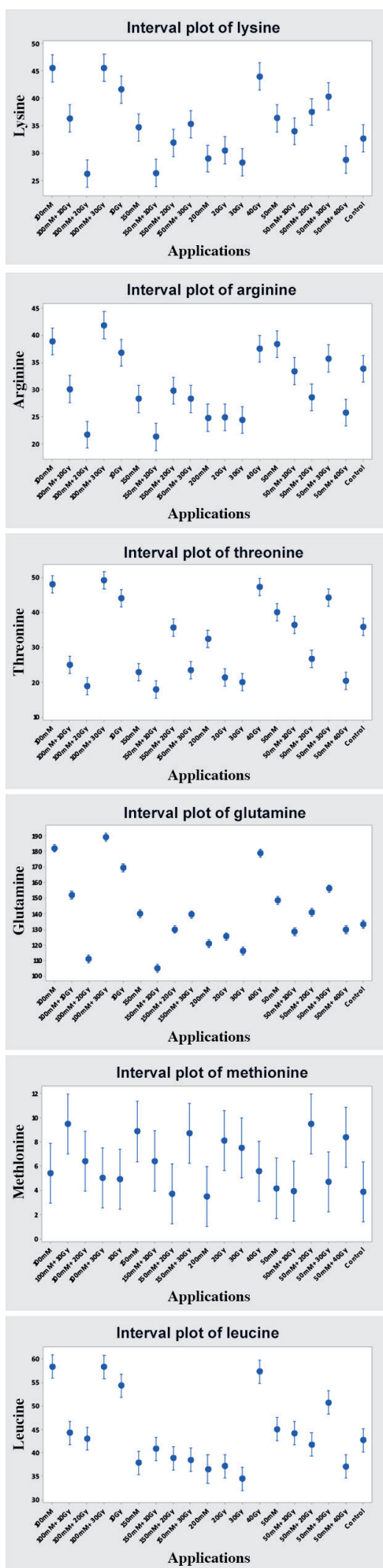
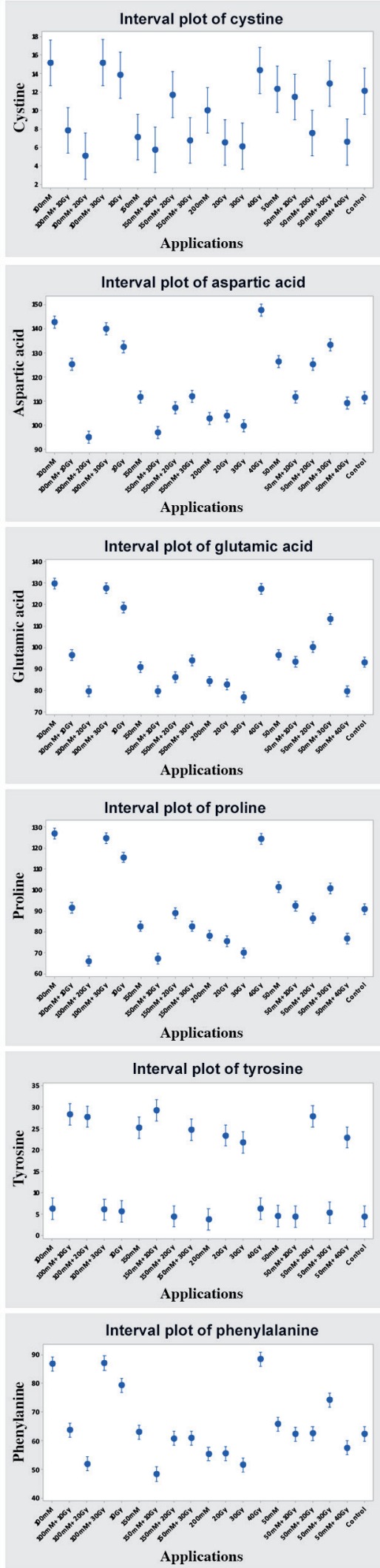
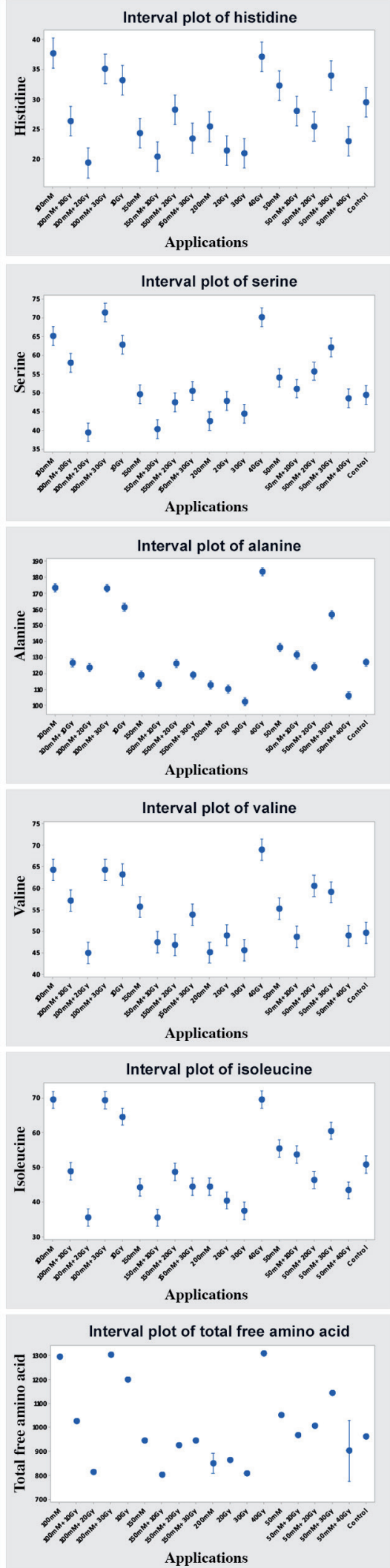
Figure 5. Interval plot of the effect of $\mathrm{NaCl}$ and low-dose gamma ray applications on the amino acid profile of common bean 'F16' (mean $\pm 95 \%$ confidence intervals).
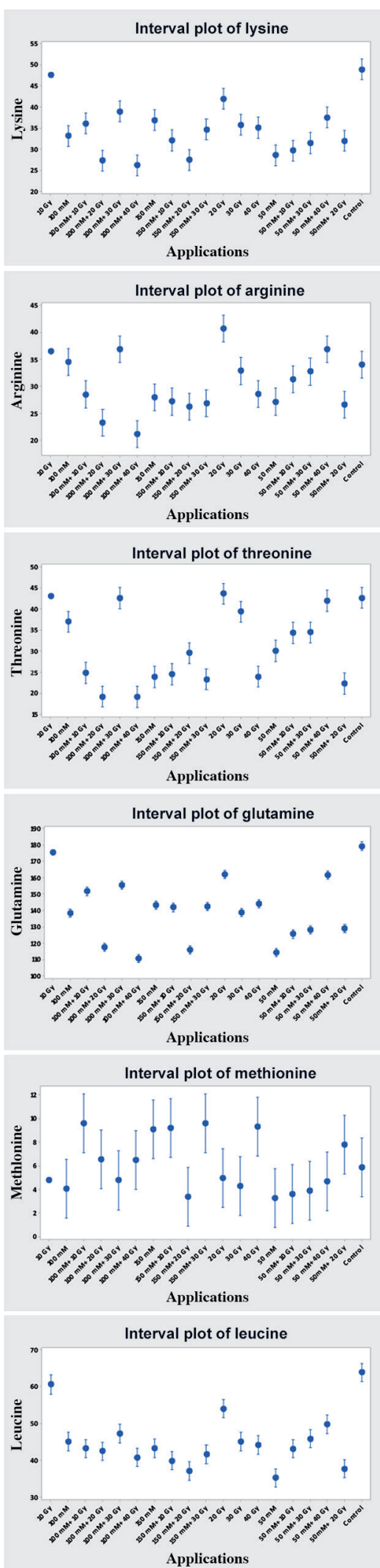
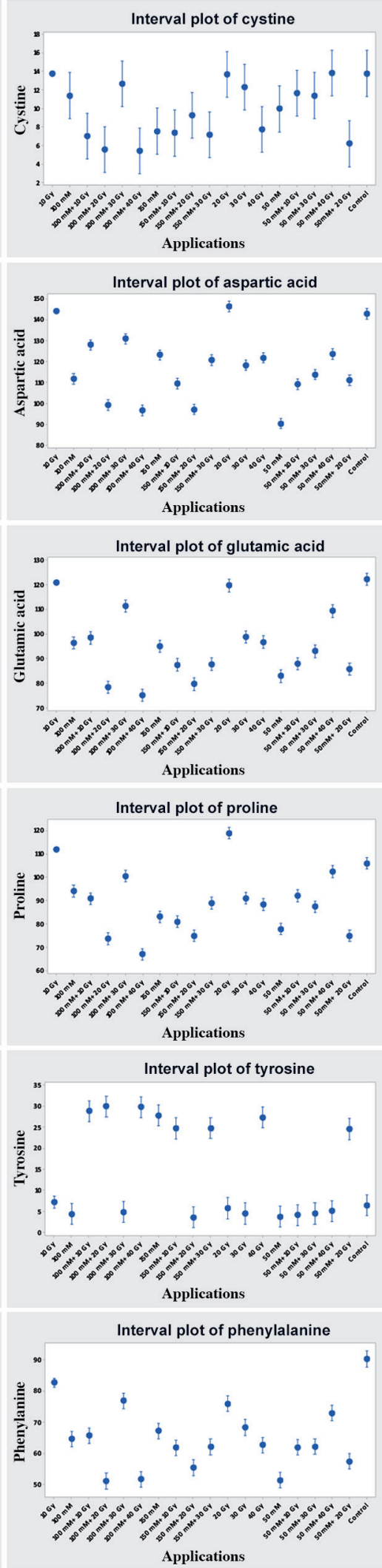

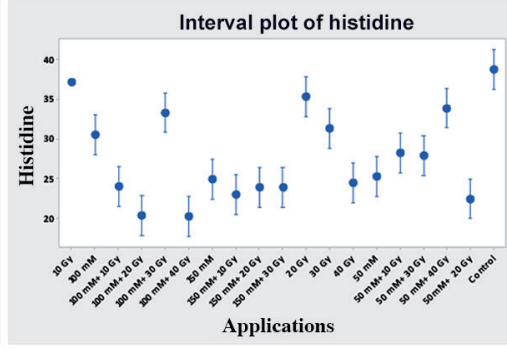

Interval plot of serine
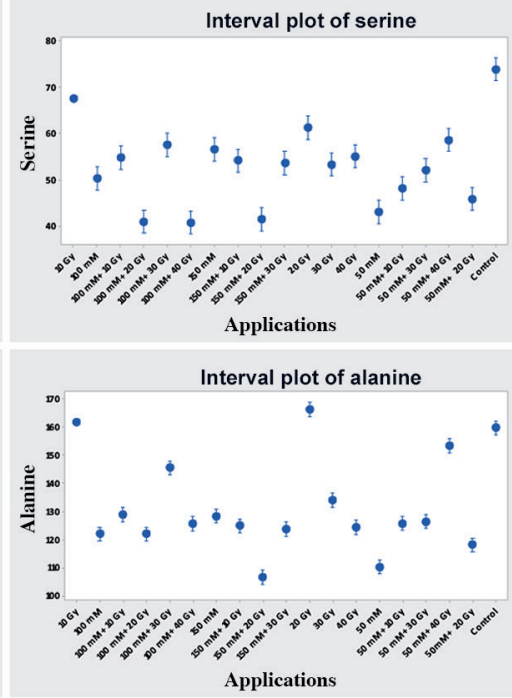

Applications
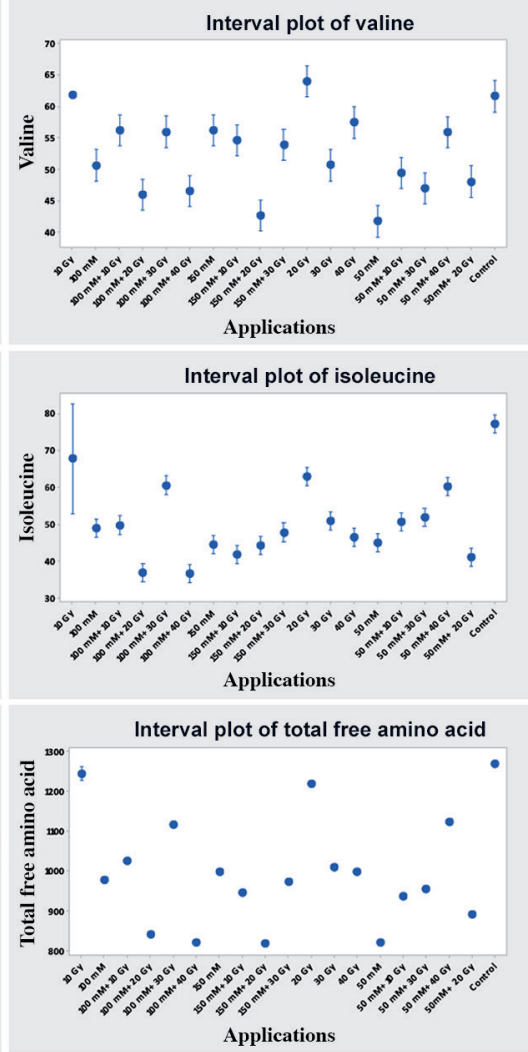
Although high gamma doses in cowpea increase chromosomal abnormalities such as anaphase delays, anaphase bridging, chromosome clustering, chromosome breaks (Dhanavel et al., 2012), low-dose gamma applications in some cowpea varieties have led to more chromosomal abnormalities (Badr et al., 2014). Chromosomal abnormalities do not always cause negative consequences. It is also reported that there is a positive correlation between chromosomal abnormalities and the increase in antioxidant enzyme activity that activates the defense mechanisms of cells (Datta et al., 2011).

Amino acids, one of the important components of plant metabolism, play various roles in growth, development and defense processes (Ali et al., 2019). Changes in the amounts of amino acids, modifying plant metabolism under stress conditions, can determine defense mechanisms in plants and help in understanding tolerance levels. From this viewpoint, the effects of gamma ray and salt stress on the amino acid profile differed between cultivars. When only low-dose gamma irradiation was applied without salt stress, in general, 40 Gy in 'Öz Ayşe' and 10 Gy in 'F16', this caused an increase in amino acid amounts. However, the increase in plant growth at some low-dose gamma rays levels is thought to be related to the stimulating effect of the gamma rays rather than the stress effect. The results for salt stress provide more explanatory information for amino acid changes. The levels of the amino acids that decreased with the application of salt stress increased with increasing stress levels. In studies conducted in soybean (Farhangi-Abriz and Ghassemi-Golezani, 2016), wheat (Aly et al., 2019a), moringa (Hussen et al., 2013), the amount of amino acids increased under salt stress, which was associated with tolerance to salt stress. However, the increase in amino acids differs according to the species and genotype. For example, Asp, Thr, Ser, Ala, Val, Gly, Ile and His were increased in moringa depending on the concentration, and in this study, there was a significant increase in the amounts of Glu, Ala, Gln, Pro, Tyr in 'Öz Ayşe' and in Tyr in 'F16'. Cavusoglu (2019) reported that Tyr, when applied externally, mitigated the negative effects of salt stress on physiological and cytological parameters. The fact that more Tyr was synthesized under severe stress may be related to the fact that Tyr acts as the precursor of several specific metabolites that perform a variety of physiological functions such as defense mechanisms, structural support, electron carrying and antioxidant activity. In this study, in both common bean cultivars, $150 \mathrm{mM}$ reached the highest value.

The fact that Ala is one of the amino acids associated with stress response (Galili, 2011) explains the increase in its amount. In wheat, Cys, Arg and Met constitute 55\% of the total free amino acids. However, Xie et al. (2020) reported that in Phragmites australis, 19 amino acids (Val, Ile, Pro, His...) accumulate depending on the salt stress period and the prolongation of the process causes an increase in the amino acid concentration. Lysine is an important signaling amino acid that regulates plant growth and responses to the environment. It is also synthesized in higher plants in a route that begins with aspartate, leading to the formation of Thr, Met and Ile (Ali et al., 2019). In this respect, when the amounts of Lys, Glu, Thr, Met and Ile were examined, they increased at $100 \mathrm{mM}$ in 'Öz Ayşe', and this increase in Met continued at $150 \mathrm{mM}$. We assume that the lack of such an increase in ' $\mathrm{F} 16$ ' is due to the sensitivity of the cultivar. In addition, the fact that the plants could not be obtained at a salt stress of $200 \mathrm{mM}$ was another evidence of the sensitivity of 'F16'. Simple organic molecules, such as free amino acids, are involved in the regulation of plant osmosis in saline soil conditions (Aly et al., 2019b). Consistent with this study, studies in different plants show that amino acid synthesis is related to the plant's stage of development, intensity, and duration of the stress (Yang et al., 2015; Farhangi-Abriz and Ghassemi-Golezani, 2016; Xie et al., 2020). The decrease of Pro after 100 mM salt application in 'Öz Ayşe' was an indication of this situation.

The amino acid contents of plants grown at salt concentrations of 50 and $100 \mathrm{mM}$ were generally highest in seeds irradiated with $30 \mathrm{~Gy}$ in 'Öz Ayşe'. The plant growth values also support this result. It is not possible to mention a single gamma rays dose for the stimulative effect of gamma irradiation at $150 \mathrm{mM}$. Since common beans are sensitive to stress conditions, gamma rays did not have a hormetic effect for highly severe stress, but low-dose gamma rays affected the amino acid profile positively under moderate stress conditions, which was also reflected in plant growth. In 'F16', 40 Gy at a salt concentration of $50 \mathrm{mM}$ had an obvious hormetic effect. In addition, the stimulating effect of $30 \mathrm{~Gy}$ at $100 \mathrm{mM}$ is generally reflected in the accumulation of all amino acids. Branched-chain amino acids (Leu, Ile and Val) accumulate during stress due to protein breakdown and can act as respiratory substrates. In Arabidopsis, a model plant, branched amino acids accumulate in response to some stress conditions such as salt, drought and herbicide application, and this accumulation is more significant than that of Pro, which has been the subject of many studies (Huang and Jander, 2017). Such an accumulation occurred for $30 \mathrm{~Gy}$ in 'Öz Ayşe' and 'F16', and the fact that these applications also promote plant growth suggests that amino acid accumulation may be due to the stimulation of amino acid synthesis rather than protein degradation. The regulatory role of amino acid metabolism in the response of plants to stress conditions includes the suppression of genes 
associated with amino acid biosynthesis and the stimulation of genes associated with amino acid catabolism in stressinduced transcriptome changes (Galili, 2011). The increase in vegetative plant growth in this study can be explained by the activation of genes that stimulate amino acid biosynthesis with a low-dose gamma rays effect.

The stability of protein synthesis in common beans and the synthesis of certain polypeptides continued under salt stress with low-dose gamma rays application (Beltagi et al., 2006). In accordance with this study, the stimulating effect of low-dose gamma rays administration at moderate salt stress was demonstrated. Similar results were obtained in a study conducted on wheat, where salt stress and gamma rays caused an increase in the amino acid content, and the authors reported changes in the protein profile depending on the irradiation dose and the salinity level (Aly et al., 2019a). In this study, since there was no external support, internal synthesis increased as a result of the stimulation by gamma rays, and tolerance to salt was achieved by stimulating plant growth. These increases are due to changes in the metabolism of free amino acids that act as osmoprotectants or to protein turnover under stress conditions. For example, in a study on Arabidopsis the free amino acid profile under water stress, as in this study, depended on the type and severity of the stress (Hildebrandt, 2018; Yobi et al., 2020). In another study, the amino acid content of beans also increased under moderate drought stress, suggesting that the amino acid level can be used as a biochemical marker in breeding studies (Andrade et al., 2016). The accumulation of amino acids indicates plant tolerance, as the accumulation of specific amino acids such as Pro, as well as secondary metabolites derived from amino acid metabolism, are associated with increased tolerance to adverse environmental conditions.

\section{CONCLUSIONS}

In this study, low-dose gamma rays stimulated vegetative growth in common beans. Amino acid accumulation in nonirradiated plants was at $100 \mathrm{mM} \mathrm{NaCl}$ in 'Öz Ayşe'. In 'F16', the control was the group with the highest amount of amino acids. This is thought to be due to the fact that ' $\mathrm{F} 16$ ' is very sensitive to salt stress. When salt stress was applied, amino acid accumulation occurred at certain gamma rays doses. Consistent with the vegetative development of plants under salt stress conditions, low-dose gamma rays have a hormetic effect at moderate salt stress, but not under severe salt stress.

\section{REFERENCES}

Ahmed, H.A.A., Şahin, N.K., Akdoğan, G., Yaman, C., Köm, D., and Uranbey, S. 2020. Variability in salinity stress tolerance of potato (Solanum tuberosum L.) varieties using in vitro screening. Ciência e Agrotecnologia 44:1-14. https://doi.org/10.1590/1413-7054202044004220.

Ali, Q., Athar, H.R., Haider, M.Z., Shahid, S., Aslam, N., Shehzad, F., et al. 2019. Role of amino acids in improving abiotic stress tolerance to plants. p. 175-203. In Hasanuzzaman, M., Fujita, M., Oku, H., Islam, M.T. (eds.) Plant tolerance to environmental stress: Role of phytoprotectants. CRC Press, Boca Raton, Florida, USA.

Aly, A.A., Eliwa, N.E., and AbdEl-Megid, M.H. 2019b. Stimulating effect of gamma radiation on some active compounds in eggplant fruits. Egyptian Journal of Radiation Sciences and Applications 32(1):61-73. doi:10.21608/ejrsa.2019.10024.1066.

Aly, A.A., Maraei., R.W., and Baraket, M. 2019a. Effects of gamma irradiation and salt stress on amino acids and protein fractions of two Egyptian bread wheat (Triticum aestivum L.) cultivars. Bangladesh Journal of Botany 48(4):1175-1184.

Andrade, E.R., Ribeiro, V.N., Azevedo, C.V.G., Chiorato, A.F.C., Williams, T.C.R., and Carbonell, S.A.M. 2016. Biochemical indicators of drought tolerance in the common bean (Phaseolus vulgaris L.) Euphytica 210(2):277-289. doi:10.1007/s10681-016-1720-4.

Aquino, K.A.D.S. 2012. Sterilization by gamma irradiation. p. 172-202. In Adrovic, F. (ed.) Gamma radiation. IntechOpen, doi: $10.5772 / 34901$.

Badr, A., El-Shazly, H.H., and Halawa, M. 2014. Cytological effects of gamma radiation and its impact on growth and yield of M1 and M2 plants of cowpea cultivars. Cytologia 79(2):195-206. doi:10.1508/cytologia.79.195.

Beltagi, M.S., Ismail, M.A., and Mohamed, F.H. 2006. Induced salt tolerance in common bean (Phaseolus vulgaris L.) by gamma irradiation. Pakistan Journal of Biological Sciences 9(6):1143-1148. doi:10.3923/pjbs.2006.1143.1148.

Calabrese, E.J. 2019. The dose-response revolution: how hormesis became significant: an historical and personal reflection. p. 3-24. In Rattan, S.I.S., and Kyriazi, M. (eds.) The science of hormesis in health and longevity. Academic Press, London, UK.

Cavusoglu, D. 2019. Physiological and cytogenetical effects of L-tyrosine in Allium cepa L. exposed to $\mathrm{NaCl}$ stress. Fresenius Environmental Bulletin 28(12A):9753-9759. 
Datta, S.K., Chakrabarty, D., Verma, A.K., and Banerji, B.K. 2011. Gamma ray induced chromosomal aberrations and enzyme related defense mechanism in Allium cepa L. Caryologia 64:388-397. https://doi.org/10.1080/00087114.2011.10589806.

De Pascale, S., Barbieri, G., and Ruggiero, C. 1997. Effects of water salinity on plant growth and water relations in snap bean (Phaseolus vulgaris L.) Acta Horticulturae 449:649-656. doi:10.17660/ActaHortic.1997.449.90.

Dhanavel, D., Gnanamurthy, S., and Girija, M. 2012. Effect of gamma rays on induced chromosomal variation in cowpea Vigna unguiculata (L.) Walp. International Journal of Current Science 12:245-250.

El-Sabagh, A.S., Barakat, M.N., and Genaidy, E.E. 2011. Towards in vitro selection studies for salinity tolerance in Canino apricot cultivar. Effect of gamma irradiation on in vitro mutation and selection for salt-tolerance. Advances in Horticultural Science 25(4):260-263. doi:10.13128/ahs-12760.

Farhangi-Abriz, S., and Ghassemi-Golezani, K. 2016. Improving amino acid composition of soybean under salt stress by salicylic acid and jasmonic acid. Journal of Applied Botany and Food Quality 89:243-248. doi:10.5073/JABFQ.2016.089.031.

Fornalski, K.W., Adamowski, L., Turowski, T.W., and Wojnarowicz, J. 2012. Search of radiation hormesis in plants: Irradiation of the cress (Lepidium sativum L.) Nukleonika 57:421-426.

Galili, G. 2011. The aspartate-family pathway of plants: linking production of essential amino acids with energy and stress regulation. Plant Signaling and Behavior 6(2):192-195. doi:10.4161/psb.6.2.14425.

Hasanuzzaman, M., Nahar, K., Alam, M.M., Bhowmik, P.C., Hossain, M.A., Rahman, M.M., et al. 2014. Potential use of halophytes to remediate saline soils. BioMed Research International 2014:589341. https://doi.org/10.1155/2014/589341.

Hildebrandt, T.M. 2018. Synthesis versus degradation: directions of amino acid metabolism during Arabidopsis abiotic stress response. Plant Molecular Biology 98(1-2):121-135. https://doi.org/10.1007/s11103-018-0767-0.

Hildebrandt, T.M., Nesi, A.N.,Araújo, W.L., and Braun, H.P. 2015. Amino acid catabolism in plants. Molecular Plant 8(11):15631579. https://doi.org/10.1016/j.molp.2015.09.005.

Huang, T., and Jander, G. 2017. Abscisic acid-regulated protein degradation causes osmotic stress-induced accumulation of branched-chain amino acids in Arabidopsis thaliana. Planta 246(4):737-747. doi:10.1007/s00425-017-2727-3.

Hussen, M.M., Lobna, S.T., Rawia, A.E., and Soad, M.M.I. 2013. Responses of photosynthetic pigments and amino acids content of moringa plants to salicylic acid and salinity. Journal of Applied Sciences Research 9(8):4889-4895.

Iglesias-Andreu, L.G., Octavio-Aguilar, P., and Bello-Bello, J. 2012. Current importance and potential use of low doses of gamma radiation in forest species. p. 263-280. In Adrovic, F. (ed.) Gamma radiation. IntechOpen, doi:10.5772/36950.

Jaipo, N., Kosiwikul, M., Panpuang, N., and Prakrajang, K. 2019. Low dose gamma radiation effects on seed germination and seedling growth of cucumber and okra. Journal of Physics: Conference Series 1380(1):1-5. doi:10.1088/1742-6596/1380/1/012106.

Kumar, D.S., Chakrabarty, D., Verma, A.K., and Banerji, B.K. 2011. Gamma ray induced chromosomal aberrations and enzyme related defense mechanism in Allium cepa L. Caryologia 64:388-397. https://doi.org/10.1080/00087114.2011.10589806.

Marcu, D., Cristea. V., and Daraban, L. 2013. Dose-dependent effects of gamma radiation on lettuce (Lactuca sativa var. capitata) seedlings. International Journal of Radiation Biology 89(3):219-223. https://doi.org/10.3109/09553002.2013.734946.

Muchate, N.S., Rajurkar, N.S., Suprasanna, P., and Nikam, T.D. 2019. NaCl induced salt adaptive changes and enhanced accumulation of 20-hydroxyecdysone in the in vitro shoot cultures of Spinacia oleracea (L.) Scientific Reports 9(1):1-10. https://doi.org/10.1038/s41598-019-48737-6.

Nimbalkar, M.S., Pai, S.R., Pawar, N.V., Oulkar, D., and Dixit, G.B. 2012. Free amino acid profiling in grain Amaranth using LC-MS/MS. Food Chemistry 134:2565-2569. https://doi.org/10.1016/j.foodchem.2012.04.057.

Qi, W., Zhang, L., Xu, H., Wang, L., and Jiao, Z. 2014. Physiological and molecular characterization of the enhanced salt tolerance induced by low-dose gamma irradiation in Arabidopsis seedlings. Biochemical and Biophysical Research Communications 450(2):1010-1015. https://doi.org/10.1016/j.bbrc.2014.06.086.

Saputro, T.B., Muslihatin, W., Wahyuni, D.K., Nurhidayati, T., Wardhani, F.O., and Rosalia, E. 2019. The variation induction of Glycine max through low dose gamma irradiation produces genetic and physiological alteration as source of tolerant variants in waterlogging conditions. Biodiversitas Journal of Biological Diversity 20(11):3299-3308. doi:10.13057/biodiv/d201124.

Vargas-Hernandez, M., Macias-Bobadilla, I., Guevara-Gonzalez, R.G., Romero-Gomez, S.D.J., Rico-Garcia, E., OcampoVelazquez, R.V., et al. 2017. Plant hormesis management with biostimulants of biotic origin in agriculture. Frontiers in Plant Science 8:1762. https://doi.org/10.3389/fpls.2017.01762.

Wang, X., Ma, R., Cui, D., Cao, Q., Shan, Z., and Jiao, Z. 2017. Physio-biochemical and molecular mechanism underlying the enhanced heavy metal tolerance in highland barley seedlings pre-treated with low-dose gamma irradiation. Scientific Reports 7(1):1-14. doi:10.1038/s41598-017-14601-8.

Wiendl, T.A., Wiendl, F.W., Franco, S.S., Franco, J.G., Althur, V., and Arthur, P.B. 2013. Effects of gamma radiation in tomato seeds. International Nuclear Atlantic Conference (INAC), Recife. 24-29 November. Associação Brasileira de Energia Nuclear (ABEN), Rio de Janeiro, Brazil.

Xie, E., Wei, X., Ding, A., Zheng, L., Wu, X., and Anderson, B. 2020. Short-term effects of salt stress on the amino acids of Phragmites australis root exudates in constructed wetlands. Water 12(2):569. doi:10.3390/w12020569.

Yang, Y., Liu, X., Jiang, Y., Xiang, Z., Xu, Q., Zhao, N., et al. 2015. Root growth, free amino acids, and carbohydrates of tall fescue in response to soil salinity. HortScience 50(4):609-614. https://doi.org/10.21273/HORTSCI.50.4.609. 
Yobi, A., Bagaza, C., Batushansky, A., Shrestha, V., Emery, M.L., Holden, S., et al. 2020. The complex response of free and bound amino acids to water stress during the seed setting stage in Arabidopsis. The Plant Journal 102(4):838-855. doi:10.1111/tpj.14668.

Zaka, R., Chenal, C., and Misset, M.T. 2004. Effect of low doses of short-term gamma irradiation on growth and development through two generation of Pisum sativum. Science of the Total Environment 320:121-129. https://doi.org/10.1016/j.scitotenv.2003.08.010.

Zaki, H.E., and Yokoi, S. 2016. A comparative in vitro study of salt tolerance in cultivated tomato and related wild species. Plant Biotechnology (Tokyo) 33(5):361-372. doi:10.5511/plantbiotechnology.16.1006a.

Zhang, B., Liu, K., Zheng, Y., Wang, Y., Wang, J., and Liao, H. 2013. Disruption of AtWNK8 enhances tolerance of Arabidopsis to salt and osmotic stresses via modulating proline content and activities of catalase and peroxidase. International Journal of Molecular Sciences 14:7032-7047. https://doi.org/10.3390/ijms14047032. 